Urologe 2012 $\cdot 51[$ Suppl 1]:5-6 DOI 10.1007/s00120-012-2981-8

Online publiziert: 19. August 2012

(c) Springer-Verlag 2012

\title{
S.C. Müller
}

Klinik und Poliklinik für Urologie und Kinderurologie, Universitätsklinikum Bonn, Bonn

\section{Wissen schafft Fortschritt und Leben}

Mit diesem Titel in leichter Abwandlung unseres Kongressmottos „Wissenschaft, Fortschritt, Leben" möchte ich betonen, dass man nur mit dem Wissen um die Probleme in der Medizin, den Status quo und die Stagnation beenden und im Sinnes des medizinischen Fortschritts auch Leben verbessern und verlängern kann.

Wir kennen sehr wohl die Probleme und Defizite in unserer studentischen Aus- und Weiterbildung und sollten rasch daran gehen diese zu lösen. Es geht um Qualitätssicherung gerade im Vergleich $\mathrm{zu}$ anderen Ländern der Welt - besonders auch auf manuell operativem Gebiet.

Wir wissen, dass das metabolische Syndrom eine globale Bedrohung gerade für jüngere Menschen in westlichen Ländern darstellt - quasi ein "Sargnagel“ der Gesundheit. Es begünstigt nicht nur die Entstehung von Tumoren, sondern eine Reihe von urologischen Krankheitsbildern wie erektile Dysfunktion, Steinbildung und Harninkontinenz. Es führt über arteriosklerotische Veränderungen zur renalen Schädigung und erhöhter Sterblichkeit. Es liegt also an uns Urologen, gerade bei jüngeren Männern präventiv tätig zu werden. Die zu diesem Kongress erstellte Präventionsbroschüre sollte als Zündfunke dienen, uns als Urologen viel mehr um den bisher vernachlässigten ,jungen Mann“ zu kümmern.

Wir wissen um die steigende Zahl von Fehlbehandlungen, die bei den Schlichtungsstellen der Ärztekammern gerade in operativen Fächern verhandelt werden und müssen alles tun, um hier für mehr Offenheit und Transparenz zu sorgen. Die in größeren Kliniken seit längerem etablierten CIRS („clinical incident repor- ting systems") müssen auch in den Praxen Einzug halten. Herr Büchler, als Vertreter der Gesellschaft für Chirurgie, wird zur Qualitätssicherung in der operativen Medizin auf unserem Kongress Stellung nehmen.

Wir wissen auch, dass unser Fach nur durch hochqualifizierte Forschung und Wissenschaft überlebensfähig ist. Hier gilt es frühzeitig Talente zu erkennen, sie $\mathrm{zu}$ fördern und Kooperationsmöglichkeiten mit anderen Grundlagenforschern zu schaffen. Wir müssen alles daran setzen, um die Qualität und Kontinuität für die Grundlagenforschung aber auch für die klinisch orientierte Forschung in unserem Fachgebiet zu steigern. Denn nur so können wir im wissenschaftlichen Wettbewerb bestehen und sowohl auf operativem als auch auf medikamentös konservativem Gebiet eine optimale und qualitativ hochwertige Versorgung unserer Patienten garantieren. All diese Fragen inklusive der Schwerpunkte aber auch der Randgebiete unseres Fachgebiets werden auf diesem Kongress diskutiert und sind im Programm- und Abstractband nachzulesen.

Mein Dank gilt den Gutachtern und der Programmkommission, die aus 721 eingegangenen Beiträgen 405 als Poster oder freie Vorträge in das Programm aufgenommen haben. Die Auswahl für die Annahme der eingereichten Beiträge erfolgte nach kritischer Bewertung durch vier verschiedene Fachgutachter letztlich durch einen unabhängigen Obergutachter der Programmkommission. Diese immense Arbeit konnte zeitgerecht nur mit Hilfe computergestützter Programme und durch die professionelle Unterstützung 
von Herrn Schinkel und Frau Scharp der Firma Pharmaservice geleistet werden. Die Hauptlast der Feinabstimmung und endgültigen Programmerstellung trugen meine Kongresssekretärin Frau Sabine Limbeck sowie Frau Andrea Chaya von der DGU-Geschäftsstelle und Frau Heike Schäfer von der Firma Interplan. Ihnen danke ich ebenso für die stets freundliche und konstruktive Zusammenarbeit wie meinen ärztlichen Mitarbeitern Herrn Dr. Rogenhofer und Herrn Dr. Strunk. Daneben waren auch alle Mitarbeiter der Geschäftsstelle der Deutschen Gesellschaft für Urologie e.V. insbesondere Frau Radziewski und Herr Petersilie immer wieder eine wertvolle Hilfe.

Die nicht minder wichtige Öffentlichkeitsarbeit in Vorbereitung dieses Kongresses gelang perfekt in enger Zusammenarbeit mit unserer immer aktiven Pressestelle in Hamburg unter Führung von Frau Bettina Wahlers und Frau Sabine Glimm. Ihnen bin ich zu großem Dank verpflichtet.

Auf dem Gebiet der Fort- und Weiterbildung hat sich die Akademie der deutschen Urologen fest positioniert. Die zahlreichen Akademieforen sind Hauptbestandteil eines furiosen Kongressbeginns am Mittwochmorgen und auch die bewährten Expertenkurse gehören inzwischen zu den stark frequentierten Veranstaltungen dieses Kongresses. Dieses Jahr haben wir mit Herrn Prof. Walsh aus Baltimore erstmals die Möglichkeit in einer interaktiven Diskussionsrunde, reserviert für Chef- und Oberärzte, aus erster Hand die operativen Tricks der offenen radikalen Prostatektomie zu erfahren. Hier möchte ich besonders dem Leiter der Akademie, Herrn Prof. Michel und auch Frau Elfy Scholten für Ihre Arbeit danken. Die Hands-on-Kurse werden diesmal vor Ort von Prof. Stolzenburg zusammen mit Herrn Prof. Rassweiler in bewährter Weise organisiert. Herr Kollege Buntrock wird zusammen mit Frau Prof. Kliesch und der GeSRU-Vorsitzenden Frau Dr. Schöne zum wiederholten Male die sehr beliebten Schülertage organisieren, um bereits einem sehr jungen Publikum klar $\mathrm{zu}$ machen was eigentlich ein Urologe ist und was er tut.

Zusammen mit allen an der Kongressorganisation Beteiligten freue ich mich Sie in Leipzig begrüßen zu dürfen. Es erwarten Sie viele spannende Themen in einem durch „Urology International“ auch weltoffenerem Kongressambiente. Leipzig als Messestadt bietet mit dem hoch attraktiven Messegelände einen idealen Rahmen für unseren Kongress. Die freundliche, überschaubare Stadt ist geprägt durch die sog. friedliche Revolution, bei der auch das Wissen und das Erkennen bestehender Probleme zu Fortschritt und besserem Leben geführt hat.

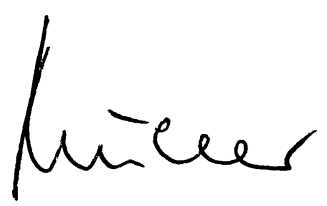

\section{S.C. Müller}

Präsident der Deutschen Gesellschaft für Urologie (DGU)

\section{Korrespondenzadresse}

Prof. Dr. Dr. h.c. S.C. Müller

Klinik und Poliklinik für Urologie und Kinderurologie,

Universitätsklinikum Bonn,

Sigmund-Freud-Straße 25, 53105 Bonn

Stefan.Mueller@ukb.uni-bonn.de 
Hier steht eine Anzeige.

黛 Springer 